\title{
Plaidoyer pour un pluralisme sous contraintes
}

Jean-Michel Berthelot

\section{OpenEdition}

\section{Journals}

Édition électronique

URL : http://journals.openedition.org/ress/532

DOI : $10.4000 /$ ress.532

ISSN : 1663-4446

\section{Éditeur}

Librairie Droz

\section{Édition imprimée}

Date de publication : 1 septembre 2003

Pagination : $35-49$

ISBN : 2-600-00878-0

ISSN : 0048-8046

Référence électronique

Jean-Michel Berthelot, "Plaidoyer pour un pluralisme sous contraintes », Revue européenne des sciences sociales [En ligne], XLI-126 | 2003, mis en ligne le 30 novembre 2009, consulté le 02 mai 2019. URL : http://journals.openedition.org/ress/532 ; DOI : 10.4000/ress.532 
Jean-Michel BERTHELOT

\section{PLAIDOYER POUR UN PLURALISME SOUS CONTRAINTES}

Ce texte fut présenté lors d'une séance d'hommages, consacrée à Jacques Coenen-Huther. Il serait paradoxal que ce qui pouvait sembler «de circonstance» en soit gommé. Ce n'est pas que l'exercice fut facile. Mais la personnalité de Jacques Coenen-Huther était, en l'occurrence, d'une grande aide. Il est en effet de ces sociologues assez rares - et à vrai dire assez exceptionnels - qui allient avec bonheur la curiosité des faits et le goût des idées, une appétence immodérée pour l'immersion dans les «terrains » les plus divers et les plus périlleux et la connaissance exigeante des classiques, le souci de la théorisation et de l'analyse et l'indépendance du point de vue et des propos. De cette attitude exemplaire, on souhaiterait pouvoir s'inspirer, pour être, aussitôt, confronté à ses propres limites : qui peut ainsi passer d'une expérience de travail dans la métallurgie de l'aprèsguerre à un long séjour dans un kibboutz de Galilée ou à une immersion profonde dans l'URSS à l'agonie? Qui peut maîtriser à ce point des références intellectuelles multiples, issues des grandes traditions nationales? Qui peut, enfin, engager, avec la sérénité toute mertonienne de l'homme de science, la discussion minutieuse des programmes les plus fondamentaux de la sociologie?

Partageant avec Jacques le souci de l'analyse et le goût de l'indépendance, j'ai opté pour une stratégie modeste: lui soumettre simplement les réflexions, auxquelles le thème qu'il avait choisi, la perception que je peux avoir de la situation actuelle de la sociologie et ma propre histoire intellectuelle, enfin, me conviaient.

De quoi va-t-il donc être question? Du pluralisme, du relativisme, de la scientificité de la sociologie, mais aussi de la prolifération des terminologies, de la pesanteur des modes, des provocations faciles, des indignations vertueuses, du vertige que provoque l'infinie cacophonie de l'empilement incessant des textes! Dans le premier cas, il s'agit de problèmes théoriques, que l'on taxe parfois, dans une vieille tradition de méfiance, d'académiques et de scolastiques, et qui ne valent souvent à ceux qui semblent s'y intéresser, que la considération distante, voire condescendante, d'une communauté pratiquant facilement la bonne conscience du «terrain»; dans le deuxième, je pense à ces réactions d'humeur, mais aussi parfois de franc découragement, dont on se sent saisi devant une discipline souvent fermée à la discussion rationnelle, clivée dans ses certitudes, ses chapelles, ses idiomes, ses vénérations et ses discrédits. 
Si la sociologie prétend être une entreprise intellectuelle de quelque qualité, elle ne peut établir cette prétention que sur pièces. Si elle veut être partie prenante du mouvement scientifique, elle doit, dans la spécificité de ses objets et de ses méthodes, décliner les traits communs à toute entreprise scientifique. GillesGaston Granger les ramenait à trois: la visée d'une réalité; l'objectif de décrire et d'expliquer; le souci de critères de validation (Granger, 1993).

Même sommaires et didactiques, ces trois critères ont un triple mérite: ils établissent une ligne de démarcation entre l'entreprise scientifique - qui vise à remplir ces trois objectifs - et des entreprises d'un autre type, idéologique, littéraire, technique, politique...; ils admettent, derrière l'unicité de la visée, une pluralité de moyens (techniques, méthodes, styles etc.); ils sont, enfin, très facilement applicables à la sociologie. Bien sûr, il n'est pas évident de dire ce qu'est la «réalité sociale», ce que sont une bonne description et une bonne explication sociologiques, ni comment fonctionne la preuve en ce domaine; mais rien n'indique que les choses soient plus simples dans les autres sciences. D'où vient alors que la question du pluralisme sociologique soit non seulement si problématique, mais si dirimante? De trois considérations:

- le pluralisme est d'abord un fait que l'on constate et qui semble irréductible: il n'y a pas d'exemple, en sociologie, de domaines où ne cohabitent, plus ou moins pacifiquement, plusieurs théories différentes;

- il est ensuite une énigme: pourquoi la sociologie, et plus largement les sciences humaines, échouent-elles partiellement à la réduction des langages et des points de vue, qui assurent le succès d'autres disciplines?

- il est, enfin, un danger: il tend, en sociologie et en ethnologie notamment, à être théorisé et institué en norme; il aboutit ainsi au relativisme radical, c'està-dire à la négation de l'entreprise de connaissance comme telle.

La prise en compte de cette question, dans l'histoire de la sociologie, me semble avoir oscillé entre trois positions: deux positions extrêmes et une position intermédiaire, ce qui ne brille pas par son originalité. Les deux positions extrêmes sont celles du monisme et du relativisme explicatifs.

La première a connu plusieurs visages. Retenons en deux:

- un visage «épistémologique »: la définition d'un idéal de scientificité unitaire, prenant comme modèle - à tort ou à raison - les sciences de la nature, affirme, en droit, la réduction de la diversité des points de vue à l'unité d'une théorie objective soumise à contrôle empirique; on trouve des prises de position de ce type chez Durkheim, Neurath ou Nagel.

- un visage «idéologique» et dogmatique: une théorie déterminée s'arroge, du fait de sa puissance interprétative et de l'extension proclamée de son domaine d'application, le statut de seule théorie scientifique fondée du domaine. Dans la mesure où, d'une certaine façon, l'ambition hégémonique peut être considérée comme la traduction juridique de la capacité explicative ${ }^{1}$, il n'est pas très étonnant que diverses théories, à un moment ou à un autre, soient tombées

On disait autrefois que les phénomènes tombaient «sous la juridiction » d'une théorie. 
dans ce travers: le marxisme, le structuralisme, la théorie du choix rationnel, etc.

L'autre position extrême est celle du contextualisme radical, qui défend la thèse que toute théorie n'est qu'une construction plus ou moins précaire, élaborée dans un contexte donné, par des agents donnés, en vue d'objectifs donnés, sans espoir d'atteindre une valeur universelle autre qu'illusoire. Bien que, d'un point de vue logique, cette position condamne à une régression à l'infini de type solipsiste - (les mots que mon ordinateur dessinent sur mon écran en ce 9 septembre 2002 à 16 h. 51 n'ont de sens que pour l'état de mon moi conscient à cet instant précis) - elle nourrit, depuis une trentaine d'années, divers programmes sociologiques et continue d'exercer un pouvoir de fascination sur de nombreux chercheurs.

Entre ces deux extrêmes, les positions intermédiaires relèvent de deux inspirations ou de deux préoccupations, que l'on pourrait qualifier de théoriques et de politiques. Sont «théoriques» les positions qui visent à réduire analytiquement la pluralité, en en faisant l'effet de points de vue fondamentaux, de décisions axiomatiques ou de programmes différents inclus dans un espace rationnel commun ${ }^{2}$; sont politiques les positions, agnostiques en leur fond, qui conçoivent la prolifération des théories comme un simple problème pragmatique de régulation de champ.

Le fil directeur de l'enquête théorique qui va suivre procède de l'évaluation suivante:

- Quelles que soient leur valeur et leur force, aucune théorie, aucun programme, à l'œuvre aujourd'hui en sociologie, n'échappent à la pertinence d'objections rationnellement fondées (Coenen-Huther, J. 2001) ${ }^{3}$ ce qui rend illusoire toute tentative de réduction radicale;

- Le relativisme, bien que refusé verbalement, dans ses formes extrêmes, est souvent accepté dans les faits, par le développement des programmes contextualistes radicaux qui l'impliquent;

- Le pluralisme de fait se démultiplie en une profusion de terminologies partielles aboutissant parfois à une sidérante cacophonie.

Ces trois jugements peuvent paraître faciles, outrés, inutiles. Il est toujours déplaisant de sembler occuper la position du censeur et toujours confortable de rappeler que cela n'empêche ni la terre de tourner, ni la caravane de passer... Et pourtant quel sociologue peut affirmer, aujourd'hui, ne pas être resté de multiples fois perplexe face à des textes, de toute obédience, dont il se demandait: «mais qu'est-ce que cela veut dire? Où veut-il en venir? Pourquoi utilise-t-il ces termes incompréhensibles ou inutiles?». Très souvent, alors que le pluralisme réglé que prônent les positions intermédiaires ne porte que sur les grands engagements théo-

Cf. d'un certain point de vue, la grande tradition théorique sociologique, de Parsons à nos jours, est entièrement définie par cette ambition, qu'elle réalise selon des accentuations diverses, qu'il s'agisse de Boudon, de Coleman, de Bourdieu, de Giddens ou de Habermas.

3 Jacques Coenen-Huther en donne un exemple dans la discussion qu'il mène dans un récent article. 
riques et programmatiques, la sociologie contemporaine propose non pas de nouvelles théories, mais de nouvelles terminologies; elle habille des contenus triviaux de termes sophistiqués, institue des métaphores en concepts et transforme la discussion théorique en ostracisme linguistique ${ }^{4}$. Contrairement à ce que l'on pourrait penser, il n'y a pas une sociologie «sérieuse» et des sociologies fantaisistes (Bourdieu invitait à se situer entre la thèse et la foutaise, - l'affaire Tessier en a proposé un audacieux dépassement dialectique): non seulement définir les critères d'une sociologie dite «sérieuse» est problématique, voire conflictuel ${ }^{5}$, mais encore, la sociologie académique et professionnelle, estampillée, des thèses, articles et rapports divers, est en permanence «contaminée» par cette dérive terminologique qui aboutit à rendre impossible ou superficielle toute discussion, faute de compréhension réciproque véritable. Je crains - car je le constate - que la réaction la plus habituelle, consiste, alors, à ne lire que les textes que l'on comprend, parce que l'on en partage le vocabulaire et les références, et à délaisser tous les autres. Or, cet état n'est acceptable - ou compréhensible - dans l'activité scientifique, qu'aux moments de crise, où le basculement d'un paradigme à un autre s'accompagne d'un remaniement terminologique en profondeur 6 . Sauf à penser que la sociologie vive une crise épistémologique permanente, il n'y a pas de raison pour qu'elle renonce à un espace de discussion commun.

Mais, il est en fait très facile de s'accommoder de cette situation: il suffit de pratiquer non pas un pluralisme théorique (justifiant et fondant la pluralité des programmes) mais un pluralisme pragmatique ${ }^{7}$ (gérant au coup par coup la situation effective de prolifération et de concurrence des terminologies). Cette deuxième option est favorisée par divers facteurs: la multiplication exponentielle des publications qui, même dans le champ étroit d'une spécialité, autorise, au mieux, leur survol; l'existence des cadres minima de référence que constituent les grandes divisions théoriques et méthodologiques classiques - plus ou moins acquises dans le cursus de formation de chacun -; le recours permanent, enfin, comme instrument de lisibilité, aux descriptions ordinaires du monde social ordinaire, que nous avons en partage. A défaut de faire progresser l'analyse sociologique, ces références communes, tant savantes que quotidiennes, assurent une communication à moindre coût et le sentiment d'appartenir à une même communauté professionnelle.

Personnellement, je trouve peu de satisfaction intellectuelle dans cette situation. Après avoir réagi contre un dogmatisme théorique dont j'avais éprouvé tant les

J'entends par-là que ne pas comprendre et utiliser tel ou tel nouveau terme est, en soi, invalidant et provoque l'exclusion de la discussion.

s Dans un lieu d'évaluation important de la discipline, en France, une discussion a opposé récemment les participants sur la question suivante: une thèse, exclusivement théorique, peut-elle être reconnue comme un travail de sociologie? Les uns soutenaient que si la thèse porte sur des questions de théorie sociologique et est de qualité, elle doit être reconnue; les autres qu'un travail qui ne comporte pas d'empirie ne peut être qualifié de sociologique.

$6 \quad$ Il n'est d'ailleurs pas assuré que la description discontinuiste que fournit Kuhn de telles crises soit la meilleure, ni la seule acceptable.

Cette situation serait encore trop simple si elle n'était pas pimentée de quelques îlots de résistance dogmatique, renaissant perpétuellement de leurs cendres pour simplifier le problème en opposant leur position à toutes les autres. 
conséquences pratiques que les insuffisances explicatives (Berthelot, 1983) et avoir essayé de trouver un fondement logico-cognitif au pluralisme sociologique (Berthelot, 1990, 1996), je pense aujourd'hui, qu'il faut aller plus loin dans un travail d'élucidation analytique, sans lequel la polyphonie naturelle d'une entreprise de pensée collective, dégénèrera de plus en plus en cacophonie. Un tel programme a, nécessairement, plusieurs aspects. J'insisterai ici sur la nécessité d'un pluralisme sous contrainte.

\section{II}

Le pluralisme, dans l'épistémologie contemporaine, est assez bien balisé. Il désigne et interroge une situation de coexistence de théories ou de programmes dans un domaine d'étude donné. Il oppose une perspective rationaliste à une perspective constructiviste. La première considère qu' in fine, existent ou se manifestent, pratiquement, des critères de différenciation entre théories concurrentes. Ces critères peuvent être formalisés (Popper, Boudon), ou entériner, de façon plus vague, le triomphe d'un paradigme - il résout davantage d'énigmes que l'ancien - (Kuhn) ou d'un programme - il continue à être heuristique quand son concurrent s'épuise - (Lakatos). L'idée commune est que les théories ou programmes différents produisent des descriptions différentes de la réalité entre lesquelles il est possible, à un moment donné, après une période déterminée, de trancher. A l'inverse, la thèse que l'on peut qualifier de constructiviste ou de contextualiste ne se contente pas d'affirmer que les théories et les programmes sont produits par des agents humains dans des circonstances données. Elle pose que les contenus ainsi générés sont incommensurables: ils engagent des ontologies différentes entre lesquelles il est impossible de trancher. Le comportement extravagant de cette personne est-il signe de possession? De dérèglement hormonal? De crise hystérique? De contamination chimique? Toutes les hypothèses valent, dira Feyerabend, tandis que le sociologue montrera comment ces croyances sont le produit d'un contexte social et culturel déterminé où elles font sens. Cette position constructiviste peut être l'objet de deux lectures, l'une mesurée, l'autre radicale:

- position mesurée: à défaut de pouvoir entrer dans l'intimité des choses, l'agent en est réduit à construire des hypothèses sur des observables; il retient celle qui est la plus cohérente avec son contexte de pensée. Elle peut être d'autant plus convaincante que, souvent, elle «marche»: l'exorciste libère la possédée. De ce point de vue, l'agent a de bonnes raisons de croire à la théorie de la possession et la prise en compte du contexte n'est pas contradictoire avec une conception rationaliste (Boudon, Raymond, 2003). Elle en permet, de surplus, la mise à l'épreuve: l'ouverture des contextes rend possible la confrontation critique des théories et assure la prééminence, à terme, des plus aptes à atteindre des mécanismes universels. Les bonnes raisons d'adhérer à la théorie de la possession sont aujourd'hui faibles, non parce que, génériquement, le contexte a changé, mais parce que, spécifiquement, ce type d'explication ne nous semble plus recevable ${ }^{8}$ comme tel (même s'il persiste dans

\footnotetext{
Bachelard, dans La psychanalyse du feu, montre que les récits de «combustions spontanées»
} fleurissent dans les textes savants jusqu'à la fin du XVIII' siècle, aussi bien en France qu'en 
certains milieux spécifiques). De ce point de vue, contextualisme et rationalisme sont donc tout à fait compatibles et le célèbre aphorisme de Feyerabend peut être interprété comme le refus de toute censure préalable dans l'élaboration des hypothèses. Bachelard parlait, lui, du passage du pourquoi au pourquoi pas pour caractériser le nouvel esprit scientifique. Mais le dernier mot appartient à la rationalité des raisons.

- Position radicale: on peut, à l'inverse, soutenir, que rien ne permet de trancher entre les diverses descriptions; que chaque agent a de bonnes raisons de soutenir son point de vue parce que c'est le sien, qu'il lui convient et qu'il exprime sa position face au réel, que c'est sa «réalité »: la possession existe parce que j'y crois et rien ne m'oblige à adopter la croyance d'autrui. Comme, cependant, pour avoir quelque crédit, une croyance doit être partagée, ce sont les conditions sociales d'un accord qui sembleront déterminantes. Le contexte, la position au sein de ce contexte, ses valeurs et références dominantes, expliqueront l'adhésion commune à une représentation partagée. Cette version radicale du constructivisme implique l'axiomatique suivante:

- la réalité est inconnaissable (elle ne tranche pas, ou ne tranche toujours qu'au sein d'une croyance $)^{9}$

- toute position est légitime, le choix entre deux positions est un problème pratique et politique

- il n’y a pas de différence légitime de point de vue entre l'analyste et l'agent ${ }^{10}$.

La sociologie est particulièrement sensible à cette position. Elle est d'abord, par vocation première, science des contextes: la spécificité de la société moderne par rapport à la société traditionnelle, d'une institution de recherche par rapport à une usine de production, d'une situation formelle d'interaction par rapport à une situation informelle, etc. définit à chaque fois un contexte différent et complexe d'action et il n'est pas nécessaire d'avoir une conception culturaliste ou déterministe de l'action pour considérer qu'un tel contexte est sociologiquement pertinent et requiert description. La sociologie est ensuite, plus peut-être que tout autre discipline, sensible à la pluralité des discours, dont le monde social lui donne à profusion des exemples à méditer.

Angleterre, pour disparaître totalement au début du XIX ${ }^{\mathrm{e}}$ siècle. Il est plus raisonnable d'y voir l'effet des transformations du mode de pensée associé au développement de la physique et de la chimie, que d'un problématique changement de contexte social et culturel.

C'est la position défendue par Bloor à travers l'exemple suivant: je donne une substance à un poulet pour obtenir un signe. Le poulet meurt. J'ai mon signe. Dans une autre croyance, la même réalité - le poulet absorbe une substance et meurt - sera traduite dans l'énoncé: la substance contenait un poison qui a tué le poulet. Selon Bloor, un énoncé ne renvoie pas à un état de chose, mais au couple indissociable état de chose + croyance. (in Sociologie de la logique, Pandore, 1981, chap. 1).

10 Cette position est adoptée par Garfinkel et l'ethnométhodologie radicale alors qu'elle est explicitement rejetée par Schütz. 
Ces deux traits concourent à opacifier les questions ontologiques, à privilégier les discours des acteurs et des institutions et à recourir aux descriptions littéraires. Certes un contexte peut être exprimé dans le langage des variables et des différences entre phénomènes identiques, relevant de contextes différents, efficacement décrits par ce moyen. C'est même le seul qui autorise la recherche d'une explication causale (jeu différentiel des variables). Malgré la tradition durkheimienne, malgré les succès indéniables de l'économétrie, cette investigation quantitative des contextes n'est pas sans poser de redoutables problèmes de construction des variables et d'interprétation des résultats, notamment dans le domaine des comparaisons internationales ${ }^{11}$. Elle nécessite des opérations de réduction de la diversité des contextes et des discours qu'ils suscitent et semble perpétuellement souffrir d'un déficit de réponse à la curiosité des lecteurs. Par réaction à ce qui paraît à certains un appauvrissement, l'étude des contextes sociaux s'est simultanément engagée dans la voie de la monographie et de l'étude de cas et a privilégié un certain type de description, associant au pouvoir de révélation que rend possible le récit, la production de catégories d'analyse inédites, oscillant entre les catégories indigènes - parties prenantes des contextes décrits et les catégories savantes - assurant la cohérence du «jeu de langage» fondant la description.

Un faisceau de raisons - dont l'attrait de la nouveauté, la sortie des sentiers battus, l'excitation de nouvelles perspectives - rend largement sensible, aujourd'hui ${ }^{12}$, au contextualisme, y compris, dans sa forme radicale. Elles ne sont pas, en soi, forcément mauvaises. Mais elles ne contraignent pas à adhérer à des positions - constructivistes, relativistes - qui oscillent entre le flou des images (construire, construction [cf. Hacking, I., 2001]) et l'aporie de leur conséquence.

\section{III}

Les angles d'attaques, pour intervenir dans cette situation, sont divers. La critique du relativisme est menée depuis plusieurs années (notamment, en sociologie, par Steven Lukes [1984] ou Raymond Boudon [1994,1995]) et semble porter ses fruits. Pour traiter plus spécifiquement du pluralisme je choisirai une autre orientation. Un des angles d'attaque possible est de réhabiliter la question

11 Cf. par exemple le courant dit de «l'analyse sociétale» dans l'analyse comparée des systèmes économiques nationaux (Maurice M., Sellier F., Silvestre J.-J., Politique d'éducation et organisation industrielle en France et en Allemagne, Paris, PUF, 1982; Maurice M., Sorge A., Embedding Organisations, Amsterdam, John Benjamin Publishing Company, 2000).

12 Cet «aujourd'hui » est sans doute partiellement faux. Il faudrait faire l'histoire du contextualisme en sociologie, depuis les monographies de l'école de Chicago jusqu'aux études de cas de la nouvelle sociologie des sciences, en passant, bien sûr, par l'ethnométhodologie. Le lien entre contextualisme, constructivisme et relativisme demanderait également à être précisé: ni Thomas, ni Berger et Luckman ne sont relativistes. Leur constructivisme (concept épistémologique) est davantage un «constructionnisme » (concept «ontologique » signifiant que la réalité sociale n’a de contenu et de sens que par les attributions symboliques qu'elle reçoit des acteurs). Cependant, il me semble que domine, aujourd'hui, une sensibilité associant contextualisme méthodologique, constructivisme épistémologique, et jeux linguistiques: on en trouverait un exemple idéal-typique dans les textes de Bruno Latour. 
ontologique en sociologie (Livet, P., Ogien, R., 2000). Réhabiliter, car elle semble avoir été moins prégnante, dans la discipline, que la question épistémologique. S'il fallait bien assurer à la sociologie un domaine qui lui corresponde (problématique de Comte et de Durkheim), ou spécifier, parmi les comportements sociaux, ceux qui relevaient d'une approche particulière (problématique de la sociologie compréhensive), ce fut davantage un travail de démarcation initiale qu'une préoccupation continue. A l'inverse, les querelles épistémologiques et méthodologiques (qui ne sont pas, évidemment, sans implications ontologiques) parcourent toute l'histoire de la discipline.

Cette prégnance de la dimension épistémologique (même dans l'affrontement entre holisme et individualisme) révèle une difficulté de fond quant à la définition de la réalité sociale et des entités, propriétés et mécanismes qui la composent. Elle désigne également la distance qu'ont souhaité prendre les sociologues avec des formes naïves de naturalisme social. Cependant, la situation générale des sciences humaines rend peut-être possible, aujourd'hui, la reprise de ces questions, auxquelles s'attache, par ailleurs, un effort renouvelé des philosophes (John Searle, Margaret Gilbert, Philippe Petitt...) Les débats de la philosophie contemporaine de l'esprit et de l'action (Fisette, D., Poirier, P. 2000) peuvent également être d'un certain secours analogique. Ils opposent deux types de programmes:

A. Des programmes réductionnistes (physicalistes, causalistes, éliminativistes) fondés sur une conception naturaliste de la réalité, comme complexe physicochimique, et de l'explication, comme action causale;

B. Des programmes non réductionnistes, acceptant la pertinence de descriptions non physicalistes (par exemple, en termes de croyances, de désirs, d'intention...) et confrontés au dilemme suivant:

B1 ces descriptions sont irréductibles et engagent des positions ontologiques variées;

B2 ces descriptions sont irréductibles, mais admettent le principe d'un monisme ontologique fondamental.

La situation est plus complexe en sociologie. Même s'il est encore difficile de décrire des états mentaux comme des croyances en termes purement physiques, personne, en revanche, ne peut nier leur lien à des dispositifs physiques déterminés. En sociologie, à moins de suivre la voie proposée par Sperber, et de réduire les institutions à des représentations collectives, celles-ci à des croyances individuelles et ces dernières à des fonctionnements cérébraux, - c'est-à-dire, de réduire la sociologie à la psychologie -, il est difficile de s'engager dans la première direction (A). Sperber (1996) lui-même ne la propose que comme un programme à développer et souligne les différences subsistant entre sociologie et psychologie, notamment aux niveaux ontologiques et descriptifs. En revanche, la voie B1, qui, pour les mêmes raisons, définit des alternatives limitées en psychologie et en philosophie de l'esprit (- celle de l'autonomie de l'esprit, par rapport aux dispositifs physiques -) ouvre, en sociologie, la véritable boîte de Pandore des entités collectives et des mécanismes inassignables. Il est donc plus raisonnable d'adopter une position de type B2, associant la pluralité des descriptions au monisme ontologique et explicatif. 
Nous définissons ainsi une première contrainte: est acceptable comme description sociologique, une description telle que les entités et les mécanismes qu'elle postule, puissent être en droit, sinon en fait, compatibles avec l'idée du monisme ontologique.

Cette contrainte est doublement discutable; elle peut être refusée; elle peut être diversement interprétée. Je ne reviendrai pas sur la première position. En ce qui concerne l'interprétation, parler de monisme ontologique n'est pas contradictoire avec l'idée de multiplicité des entités, des propriétés, des mécanismes. D'un certain point de vue, en sciences sociales comme ailleurs, on avance aussi en « découvrant» de nouvelles entités, de nouveaux mécanismes: la kula, le potlatch, les groupes de pressions, les cols blancs, les zones d'incertitude, les acteursréseaux, les cités, l'aliénation, l'étiquetage, l'accountability... Mais ces entités sont des étiquettes théoriques: on ne peut pas rencontrer le potlatch, comme on pensait, au XVI ${ }^{\mathrm{e}}$ siècle, pouvoir rencontrer le «monstre marin habillé en moine ${ }^{13}$ ou, au Moyen Age, une licorne... On ne peut que saisir des comportements manifestant, entre des actants déterminés, des échanges ordonnés, c'est-à-dire soumis à une règle. La spécificité de cette règle, l'extension de son pouvoir dans des communautés déterminées, sa stabilité dans le temps, invitent à en faire un type particulier d'échange et à lui donner le statut d'institution ${ }^{14}$ : $\operatorname{actants}^{15}$, objets, règles, échange, institution sont une sorte de premier «précipité » de cette réduction. Il est possible d'aller plus loin et d'introduire des termes comme l'intentionnalité, l'interaction, la contrainte, les sentiments moraux, la culture, les rapports de domination, les valeurs symboliques (associées aux objets échangés, aux gestes, aux attitudes...). On pourrait, très grossièrement, décrire ainsi cette situation théorique. La diversité du donné est celle des ontologies ordinaires (ou spécialisées), c'est-à-dire des entités et propriétés manipulées dans la vie de tous les jours et dans les sphères déterminées d'activité: un cuisinier, un magasinier, un maçon, un prêtre, un voyant... ont affaire, dans leur activité professionnelle, à un univers défini d'objets, de propriétés, de marques sociales, de règles qu'ils ont en partage avec ceux qui participent de cette même sphère. Le travail ontologique du sociologue est d'opérer un triple mouvement:

- de repérage et de description des entités propres à un contexte déterminé;

- de réduction de ces entités à des catégories et des mécanismes plus généraux (acteurs, règles, institutions, sentiments moraux, raisons, interactions...);

13 Très sérieusement décrit par le naturaliste Conrad Gesner.

14 Jacques Coenen-Huther fournit un exemple magnifique de cette situation dans son étude des «files d'attentes» en URSS où la durée exceptionnelle de l'attente - de plusieurs heures à plusieurs jours - contraint à l'élaboration de règles particulières de substituabilité (je remplace mon voisin pour lui laisser le temps d'aller faire autre chose) et de réciprocité (lui - ou un autre fera de même pour moi.) («Les files d'attente en Russie soviétique», Revue Française de Sociologie, 1992, vol. XXXIII, t. 2, repris in Observation participante et théorie sociologique, Paris, L'Harmattan, 1995).

15 Je n'utilise pas ce terme au sens de la sémiologie structurale, mais comme concept minimal (support d'action), en opposition aux concepts habituels, déjà déterminés, «d'agent» ou «d'acteurs ». 
- d'interrogation sur la congruence de ces derniers avec l'état d'une ontologie fondamentale que peuvent dessiner les sciences du vivant et de la nature.

Dans ce mouvement - et de façon toujours aussi schématique -, l'enjeu est le deuxième niveau, que l'on pourrait, pour simplifier, qualifier d'ontologie sociale. Il désigne les entités, les propriétés, les mécanismes que l'on peut, spécifiquement qualifier de sociaux. Le troisième niveau est celui d'une ontologie générale, où sera, par exemple, interrogé le statut de l'intentionnalité, de la norme, etc. La première contrainte peut donc s'exprimer ainsi: clarifier les implications ontologiques de ses descriptions; veiller à leur conformité avec des mécanismes physiquement, biologiquement, psychologiquement assignables; thématiser et soumettre à interrogation les éventuelles distorsions ou bifurcations. Le monisme ontologique ne signifie rien de plus. Il est cependant une contrainte bien réelle. Donnons-en un exemple simple: les phénomènes d'influence idéologique sont très largement utilisés pour rendre compte, partiellement ou totalement, d'une position ou d'un comportement; il peut s'agir des effets de la violence à la télévision, de ceux du patriotisme cocardier dans les manuels scolaires ou de l'influence de la pensée libérale sur les comportements des entrepreneurs, ou de celle de la phénoménologie dans l'œuvre de Bourdieu, etc. Le mécanisme commun (influence d'un complexe déterminé de représentations sur une catégorie définie d'acteurs) relève du niveau 2 ci-dessus. Pour qu'il soit admissible, la règle posée implique que soient interrogées ses conditions de possibilité effective dans notre monde $^{16}$. L'influence nécessite qu'il y ait eu contact attesté, défini, voire mesuré (on ne « lit pas » la pensée libérale, mais tel ou tel texte; s'il n'y a pas eu lecture, il faut des relais par laquelle cette pensée - à déterminer - est transmise; l'effet d'un contact ponctuel n'est pas le même que celui d'un commerce répété, etc.); elle nécessite également que la corrélation entre l'élément «influant» et le comportement «influencé», puisse être établi sans ambiguïté; qu'il ne puisse pas être attribué à une autre cause; que le mécanisme psychologique de l'influence, enfin, soit congruent avec ce que l'on peut savoir du fonctionnement de l'esprit.

Cette première contrainte est fortement liée au règne de la «pluralité des jeux de langage » ou des «descriptions multiples », qui régit la transcription des phénomènes sociaux. J'utiliserai là deux guides. Gilles Gaston Granger (1982) oppose les «faits actuels» aux «faits virtuels»; les premiers sont, littéralement, inépuisables et peuvent être décrits à l'infini. Les seconds sont des faits schématiques, arrachés à leur contextualité historique, et insérés dans les termes d'un modèle théorique. Elisabeth Anscombe (2002), dans sa recherche d'une description de l'action intentionnelle, propose, de fait, une procédure de réduction de la pluralité des descriptions possibles. Décrire, en tant qu'action intentionnelle, l'action d'un individu qui bouge son bras (A), active ainsi une pompe (B) et remplit une citerne d'eau empoisonnée (C), qui entraînera la mort des habitants d'une villa (D) et l'échec du mauvais coup qu'ils préparaient (E), implique une réduction successive des descriptions vraies possibles de l'action A (bouger le bras): élimination

C'est-à-dire dans un monde qui, à la différence de ceux de la science fiction, ignore, par exemple, l'influence psychique à distance ou la prise de contrôle immédiate et totale du comportement d'une entité par une autre, etc. 
des descriptions purement mécaniques et (non intentionnelles), élimination des descriptions des circonstances de A non associées à l'enchaînement A-B-C-D-E (par exemple bouger le bras en sifflant, ou sur un rythme particulier), élimination des descriptions impliquant des éléments mentaux extérieurs à l'enchaînement AB-C-D-E (je bouge le bras pour m'amuser, pour faire circuler le sang, pour gagner ma vie), élimination de l'épisode E (qui n'est qu'une conséquence induite et non une action vérifiable, comme la mort des habitants). La réponse est alors : 1'action intentionnelle tient dans l'enchaînement des quatre phases A, B, C, D et l'intention n'est rien d'autre que cette action. Ces deux références (Granger, Anscombe), nous fournissent en quelque sorte les bornes de l'espace (fait actuel / fait virtuel) et un modèle de construction d'un chemin allant de l'une à l'autre (la réduction des descriptions). Ce chemin est défini par la visée de l'analyste: pour reprendre l'exemple d'Anscombe, il sera différent selon que l'on s'intéresse au problème théorique de l'intention de l'acte, au problème moral de la responsabilité de l'homme actionnant la pompe, ou au problème physico-chimique de la dilution, de la diffusion et de l'efficacité des molécules de poison.

Le pluralisme des «jeux de langage» ou des «descriptions multiples» admet donc deux limites: la première est qu'il doit s'agir de «descriptions vraies » et non, farfelues ou putatives (Anscombe rejette ainsi de la description le chaînon $\mathrm{E}$ et ses autres conséquences possibles). La deuxième est que les descriptions doivent être ordonnées à un objectif de pensée. En d'autres termes, elles doivent être pertinentes.

Est ainsi pointée une deuxième contrainte: une description acceptable en sociologie doit être une description sociologiquement pertinente. Cela semble être une lapalissade et le devient effectivement si la sociologie est définie de façon exclusivement contextualiste, comme ensemble des travaux adoptant à un moment donné l'étiquette sociologique. Cela ne l'est plus si des critères différentiels sont proposés. Malgré la diversité des positions possibles, la tradition sociologique nous permet de ne pas être totalement démunis en l'affaire. Trois critères me semblent pouvoir être retenus:

1. Celui de la visée cognitive: la sociologie peut être assignée à des fins diverses et comprendre en son sein, des infléchissements notables : témoignage, dénonciation, critique, restitution littéraire, expertise... Ces diverses formes ne peuvent cependant prendre un sens scientifique et disciplinaire que dans le postulat d'un noyau théorique - effectif ou à construire - visant à fournir l'intelligibilité des phénomènes et situations décrits en les rattachant à des entités, des propriétés, des mécanismes de plus grande généralité.

2. Si nous appelons «modèles d'intelligibilité» les constructions de ce niveau, ceux-ci doivent être spécifiques: ils doivent relever d'un type de description que la tradition a résumé dans l'aphorisme: «expliquer le social par le social». Les comportements décrits doivent être des manières de faire dont l'élucidation échappe au simple déterminisme physique ou biologique et relèvent d'une causalité sociale.

3. Cette causalité sociale peut renvoyer, dans la tradition sociologique, à des mécanismes également divers: effets d'imposition culturels et institutionnels, 
effets structuraux, effets dialectiques, effets d'interaction, effets émergents, effets symboliques... Néanmoins, deux éléments sous-jacents définissent l'espace de contrainte d'une explication recevable: elle doit pouvoir intégrer les raisons des agents - aussi bien dans leur diversité de registre que dans l'écart possible entre raisons affichées et raisons effectives -; elle doit pouvoir s'inscrire dans une chaîne causale concrète, en droit - sinon en fait - reconstructible.

Face à l'extrême diversité des descriptions du social, il est clair que les deux contraintes que nous proposons, sont, dans leurs termes et leur acception même, discutables. Elles ont cependant l'intérêt de baliser autrement le champ du pluralisme sociologique. Elles sont solidaires, enfin, d'une troisième contrainte non moins essentielle quoique plus opératoire et pragmatique.

Le pluralisme est réduit, au sens où sa surface d'effectivité est ramenée, par le jeu des deux contraintes, à un périmètre défini. Mais au sein de ce périmètre subsiste la diversité, tant ontologique qu'explicative: il est ainsi possible, par exemple, de n'admettre aucune entité collective; mais il est tout aussi possible d'en admettre certaines - les «organisations», les «communautés professionnelles », les «partis politiques », etc. dès lors que les contraintes une et deux sont respectées. De même l'idée de «causalité sociale » ne préjuge pas des mécanismes effectifs par lesquels elle se réalise: pour les systémistes cela passe nécessairement par des formes de régulation, assurant l'homéostasie du système décrit; pour les actionnistes, les raisons des acteurs sont les causes de leur comportement, etc.

Le pluralisme subsiste donc. Mais le contrôle auquel il se soumet invite à le gérer autrement. Spécifiquement, il requiert que la simple gestion politique et pragmatique, expression de la coexistence pacifique et l'ignorance mutuelle, soit remplacée par une gestion argumentative, fondée sur l'idée d'un espace commun de discussion et d'analyse. Cela implique une troisième contrainte, que l'on pourrait appeler contrainte analytique de commensurabilité.

La thèse de l'incommensurabilité des descriptions multiples s'élabore sur l'arrière-fond d'un double mécanisme à l'œuvre au sein des disciplines scientifiques : la réduction et la rupture. La réduction vise à ramener la pluralité des perspectives possibles à un modèle unifié épuré, assurant la construction, la confrontation et l'accumulation de «faits virtuels»; la rupture se manifeste par l'adoption d'un nouveau système terminologique, récusant la pertinence des systèmes antérieurs ou concurrents et proposant, littéralement, un nouveau monde.

Les raisons qui peuvent amener à préférer une description à une autre sont évidemment diverses: différence d'entités et de mécanismes explicatifs retenus, d'échelles d'observation privilégiées, d'expériences restituées, de liens tissés avec des modèles transdisciplinaires, d'ouverture à un mouvement de réflexion plus large - comme celui des systèmes symboliques à une époque ou de la cognition sociale aujourd'hui. Rien cependant ne permet de penser que, par définition, ces descriptions s'excluent mutuellement, a fortiori qu'elles produisent des mondes différents. $\mathrm{Si}$, inversement, on adopte le principe qu'elles parlent $\mathrm{du}$ même monde (contrainte 1) et qu'elles visent à le rendre intelligible par la discipline concernée (contrainte 2) la seule question est celle de la pertinence relative 
de chacune. Cette question peut être envisagée de deux façons, parfaitement compatibles:

1. La première consiste, dans une version modeste, à élaborer des critères de confrontation différentielle entre théories et, dans une version ambitieuse, à proposer une synthèse des principes déterminants des diverses approches. Force est de constater que si la version modeste fonctionne pour des théories admettant des prémisses ou des axiomes communs, dans le cas contraire, la confrontation ne s'opère pas et chacun continue de camper sur ses positions (Boudon, R., 1971). La version ambitieuse, pèche, à l'inverse, d'un excès d'ambition et d'une déficience de discussion. Sa prétention implicite - ou explicite - à résoudre des problèmes ontologiques et épistémologiques par ailleurs insuffisamment débattus, discutés et thématisés, transforme l'effort des chercheurs en une sorte de posture d'auteur - celle de Parsons, de Giddens, d'Habermas, de Bourdieu, de Boudon... - finalement plus révérée ou rejetée, que discutée en termes programmatiques.

2. La seconde voie est plus collective: elle prend acte du développement multiple et contrasté des sciences sociales, de la nécessaire division des tâches et des intérêts qui s'est opérée en leur sein, pour pointer un lieu à la fois crucial et trop peu défriché: celui de l'analyse comparée des grands programmes, des grandes théories et de leurs arrière-fonds ontologiques et épistémologiques.

La troisième contrainte que nous semble devoir respecter aujourd'hui le pluralisme en sciences sociales, pour passer de la cacophonie à une réduction féconde, réside donc dans l'acceptation de règles de travail et de confrontation, orientant aussi bien les diverses descriptions proposées que l'ouverture à des modèles disciplinaires extérieurs. Ces règles ne peuvent évidemment pas être imposées ni administrativement, ni même théoriquement. Elles ne peuvent se construire, comme habitus disciplinaire, que dans la pratique de l'échange, de la discussion, de l'évaluation.

Cependant, face aux clivages factices ou ritualisés, que nous avons évoqués plus haut, on peut, pour finir et à titre de proposition de travail, proposer le chantier suivant. Dans chaque cas, il s'agit de règles, parfois présentes, implicitement ou explicitement, mais jamais constituées, dans leur totalité, comme un corpus partagé et contraignant.

- Règles communes régissant le travail au sein des divers programmes, des diverses théories et des diverses approches:

- Règle d'économie ontologique: ne pas introduire d'entités ou de mécanismes inutiles, occultes ou implicites; ne pas introduire de terminologies nouvelles qui ne soient pas fondées sur une effective révision ou sur une effective découverte.

- Règle de confrontation épistémologique: poser un principe de justification, rationnellement partageable, c'est-à-dire fondé sur un principe commun de la preuve et des équivalences empiriques aux propositions avancées. 
- Règle de cohérence syntaxique: respecter les contraintes syntaxiques du programme ou de la théorie utilisés, de façon à ne pas conforter, au titre d'exemples, une position en introduisant des descriptions relevant d'un autre registre.

- Règle de réflexivité: s'appliquer les contraintes et les règles précédentes à soi-même.

- Règles de confrontation entre programmes:

- Rechercher les compatibilités et les incompatibilités entre les cartes ontologiques, les systèmes syntaxiques et les règles de validation des divers programmes.

- Gérer les proximités en mettant à l'épreuve des modèles de réduction, susceptibles d'intégrer des théories et des programmes différents.

- Gérer les oppositions en clarifiant les points de bifurcation, leur nature et leurs enjeux; en élaborant une hiérarchie du raisonnable; en proposant des observations ou des expériences de mise à l'épreuve différentielle.

- Articuler la progression de la réflexion rendue ainsi possible avec la prise en compte du social dans les disciplines voisines et dans la philosophie des sciences sociales.

Terminer par un tel programme, j'en ai conscience, peut paraître à ce point utopique, irréaliste, ignorant de «la science telle qu'elle se fait», que j'ai scrupule à le présenter. En général, on tolère une telle naïveté chez les jeunes chercheurs, mais l'âge est censé détruire les illusions et faire renoncer à l'idéal d'une science désintéressée et fondamentale. Je ne dirai, pour ma défense, qu'une seule chose. Si le mythe d'une transformation heureuse de la société grâce aux sciences sociales a été fortement écorné, il est une tâche qui, non seulement demeure la leur, mais est peut-être la seule à justifier leur existence: celle de produire de la réalité sociale des descriptions suffisamment éclairantes et justes pour participer à l'effort de compréhension et de gestion de la réalité qui détermine de plus en plus notre devenir commun. Je suis loin d'être sûr que, individuellement et collectivement, nous pouvons avoir le sentiment que la sociologie remplit bien, voire honorablement, ce contrat aujourd'hui. Or celui-ci ne relève pas d'une pensée providentielle mais d'un effort partagé dont les quelques pistes ici défendues ne visent qu'à proposer une voie commune possible.

Université de Paris-Sorbonne (Paris-IV)

\section{RÉFÉRENCES BIBLIOGRAPHIQUES}

Anscombe, Elisabeth (2002), L'intention, Paris, Gallimard (Ed. anglaise 1957).

Berthelot, Jean-Michel (1983), Le piège scolaire, Paris, PUF.

Berthelot, Jean-Michel (1990), L'intelligence du social, Paris, PUF.

Berthelot, Jean-Michel (1996), Les vertus de l' incertitude, Paris, PUF. 
Bloor (1981, chap. I), Sociologie de la logique, Pandore.

Boudon, Raymond, (1971), La crise de la sociologie, Genève, Droz.

Boudon, Raymond (1994), «Les deux sociologies de la connaissance scientifique», in Boudon R. et Clavelin M., Le relativisme est-il résistible?, Paris, PUF, pp. 17-41.

Boudon, Raymond (1995), «Sur quelques aspects du relativisme contemporain», in Boudon R., Le juste et le vrai, Paris, Fayard.

Boudon, Raymond (2003), Raison, bonnes raisons, Paris, PUF.

Coenen-Huther, Jacques (1992, 1995), «Les files d'attente en Russie soviétique », Revue Française de Sociologie, 1992, vol. XXXIII, t. 2; Observation participante et théorie sociologique, Paris, L'Harmattan, 1995.

Coenen-Huther, Jacques (2001), «Sens moral ou raisons fortes?», L'Année sociologique, 2001, n 51, pp. 233-254.

Fisette, D., Poirier, P. (2000), Philosophie de l'esprit. Etat des lieux, Paris, Vrin.

Granger, Gilles-Gaston (1992), La vérification, Paris, O. Jacob.

Granger, Gilles-Gaston (1993), La science et les sciences, Paris, PUF, «Que-sais-je?».

Hacking, I. (2001), Entre science et réalité. La construction sociale de quoi?, Paris, La Découverte.

Livet, P., Ogien R. (2000), L'enquête ontologique. Du mode d'existence des objets sociaux, Raisons pratiques, $\mathrm{n}^{\circ} 11$, Paris, EHESS.

Lukes, S. (1984), «Relativism in its Place», in Hollis M. et Lukes S. (ed.), Rationality and Relativism, Cambridge, Massachusetts, The MIT Press, $2^{\circ}$ éd. 1984, pp. 261-310.

Maurice, M., Sellier, F., Silvestre, J.-J. (1982) Politique d'éducation et organisation industrielle en France et en Allemagne, Paris, PUF.

Maurice, M., Sorge, A. (2000), Embedding Organisations, Amsterdam, John Benjamin Publishing Company.

Sperber, D. (1996), La contagion des idées, Paris, Odile Jacob. 